\title{
Effect of Transcranial Direct Current Stimulation and Reaction Time Training on Cognition and Choice Reaction Time in Recreational Soccer Players (FAST Trial): A Protocol for A Randomized Clinical Trial
}

Eduaro de Moura Neto ( $\square$ eduardonetofisio@gmail.com )

Universidade Federal do Triangulo Mineiro https://orcid.org/0000-0003-0055-0822

Gustavo José Luvizutto

Universidade Federal do Triangulo Mineiro

Luciane Aparecida Pascucci Sande de Souza

Universidade Federal do Triangulo Mineiro

Study protocol

Keywords: Transcranial direct current stimulation, anodal tDCS, reaction time, cognition, soccer players

Posted Date: September 28th, 2020

DOI: https://doi.org/10.21203/rs.3.rs-30771/v1

License: (c) (1) This work is licensed under a Creative Commons Attribution 4.0 International License.

Read Full License 


\section{Abstract}

Background: Studies examining the effects of transcranial direct current stimulation (tDCS) and reaction time (RT) training on choice RT in the lower limb during a specific task linked to soccer practice, such as kicking, are lacking. Accordingly, this proposal aimed to evaluate the efficacy and safety of tDCS and RT training in choice RT and cognitive function during recreational soccer.

Methods: In this single-center, randomized, placebo-controlled, double-blind, parallel-group study of 30 recreational soccer players, tDCS will be applied in the primary motor cortex (M1). Participants will be divided into (1) anodal tDCS plus RT training and (2) sham tDCS plus RT training. The investigator will be blinded to patient treatment before the first session, last session, and 1 day after the last tDCS session. In total, 15 sessions will be performed using a schedule of three times a week for 5 weeks. The RT training protocol will be delivered during tDCS and will comprise kicking a ball from a platform, placed on either the right or the left side, depending on a light cue, during a 10-min interval (between minute 5 and minute 15 after 20-min stimulation). Changes in choice RT in the upper and lower limbs will be considered primary outcome measures, and changes in Trail Making Test and Digit Span Test will be considered secondary outcome measures. Any adverse effects during the study period will be reported. To detect changes in continuous outcomes, a the anodal tDCS and the sham mode will be compared using a t-test for normal distribution, or the nonparametric Mann-Whitney test for non-normal distribution; changes will be considered significant at $p<0.05$.

Discussion: The FAST trial is a new proposal that uses a novel method of evaluation and training of choice RT in soccer players through a functional task. The anodal tDCS along with choice RT training with a functional task can be an efficient tool for athletes to prevent injuries. Our findings may be useful for soccer athletes to have enhanced responses during a game, and to improve performances in championships.

Trial Registration: Registro Brasileiro de Ensaios Clínicos (Rebec) - RBR-9bjttc

Date of registration: 02/28/2020

Last Update: 04/20/2020

\section{Introduction}

Soccer athletes must utilize the cognitive ability to select and perform actions in response to different stimuli during a soccer game [1]. Soccer environments are unique, dynamic, and often time-constrained, where cognitive-perceptive skills used to enable the best action during the game can be assessed [2-4].

One important variable to assess cognitive function in soccer athletes is reaction time (RT) [5]. It has been shown that the auditory RT is less important than the visual RT, whereas the choice RT is more important, as soccer is very much a visual game due to the demands of constant visual tracking of the ball and 
players in motion [6]. Ando et al. (2001) [7] analyzed the difference between the RT of athletes and nonathletes using a computer to control visual stimuli. In their findings, it was observed that athletes have shorter RTs compared to non-athletes, showing the importance of sports practice on cognitive function.

There are several ways to decrease RTs, either through visual motor training [8] or through the use of noninvasive brain stimulation, with transcranial direct current stimulation (tDCS) being the most common [9]. tDCS is a noninvasive brain stimulation technique that can be safely and easily applied to healthy individuals [10]. In addition, tDCS stimulation may increase cortical activation in humans and influence motor function. Stimulation can be achieved by increasing cortical (anodic) activity or by decreasing cortical (cathodic) activity [11]. Anodically in the primary motor cortex (M1) has the ability to improve performance in simple tasks by lowering the RT [12-15].

Stimulation of the M1 area in healthy individuals, to lower limbs during simple motor tasks associated with RT training, is limited to a single study. Tanaka et al. (2009) [16] conducted a survey of healthy individuals who were instructed to press a pedal while observing a visual stimulus. The study results showed no significant decrease in lower limb single RT after anodic tDCS. Most RT studies in soccer players are associated with upper limb triggering, but only one study evaluated the effect of the tDCS on the RT of soccer players, but the specific reaction time of the kick was not analyzed [17].

According to published literature, there are no studies that have determined the effects of the tDCS and RT training on choice RTs in the lower limb during a specific task linked to soccer practice, such as kicking.

The principal aim of this study is to evaluate the efficacy and safety of tDCS and RT training in choice RTs and cognitive function during recreational soccer. We hypothesize that tDCS in conjunction with RT training will reduce choice RT in the lower limbs and will improve cognitive function in the participants.

\section{Methods}

\section{Design}

This single-center, randomized, placebo-controlled double-blind, parallel-group study of 30 recreational soccer players will be conducted in accordance with the Consolidated Standards of Reporting Trials (CONSORT) 2010 flow diagram [18] (Figure 1).

\section{Sample size}

We estimate a minimum sample size per group of 15 , assuming the following parameters: simple random sampling; type I and II error probabilities equal 0.05 and 0.20 , respectively; the absence of confounders; normal distribution of the outcome "percentage of deviation" with a mean baseline equal to a $15 \%$ reduction in the outcome after $\mathrm{tDCS}$; an estimated reduction of between $8 \%$ and $3 \%$ for the sham group; and a variation coefficient of $20 \%$. The sample size calculation was performed using G*Power 3.1.3 (Franz Faul, University of Kiel, Kiel, Germany). 


\section{Location, setting and recruitment}

All assessments will be conducted at the Federal University of Triangulo Mineiro within the biomechanics and motor control laboratory in Uberaba, Brazil. Patients will be recruited by direct contact from the researcher or invitation via social networks and selected according to the inclusion and exclusion criteria. The included patients will be randomly divided into two groups in a 1:1 ratio with a list of random numbers (that will be generated by the researcher).

\section{Participants}

\section{Inclusion criteria}

Neurologically healthy individuals with the following characteristics will be recruited from the community, mainly through the use of social media: aged 18-30 years, male, right-handed or left-handed with no associated comorbidities such as orthopedic, neurological, or visual problems.

\section{Exclusion criteria}

Individuals will be excluded if they present with any metal-in-cranium injuries near the electrode placement area, a cardiac pacemaker in situ, intracerebral vascular clips, or any other electrically sensitive support system, epilepsy, severe cognitive impairment, previous visual disturbances, or depression with scores $>8$ in the Hospital Anxiety and Depression Scale (HAD).

\section{Procedures (template)}

The participants, after being recruited from the community and social media, will sign a consent form. All participants will be instructed to perform cognitive scales, such as the Trail Making Test and Digit Span Test, and will be asked to answer the HAD to check for exclusion criteria for the tDCS application. After screening, participants will be randomized into the following two groups: (1) anodal tDCS plus RT training and (2) sham tDCS plus RT training. The investigator will be blinded to the treatment that the patient receives before the first session, last session, and 1 day after the last tDCS session (Table 1). At the end of all sessions, participants will be instructed to respond to a questionnaire evaluating any adverse effects.

\section{Randomization and blinding}

The concealed randomization schedule will be established using a computer-generated random number sequence, and maintained by an offsite investigator who will neither be involved with the enrollment nor with the assessment of study participants. A second research assistant will sequentially open consecutively numbered, randomly ordered, opaque envelopes containing the group allocation (in a 1:1 ratio) after the baseline assessment. All participants will receive RT training for 10 min, three times a week, for 5 weeks during the 20 minutes of tDCS application. The tDCS will be applied three times a week 
for 5 weeks for a total of 15 sessions, wherein the patient will be evaluated before the beginning of the first session, last session, and 1 day after the last session by a blinded investigator.

\section{Intervention}

The tDCS will be applied in accordance with the technique used by Seidel and Ragert (2019) [17]. The participant will be placed in a room with minimal external influences (such as noise, lamps, or electromagnetic waves), and positioned in a standing position at the front of the platform. For stimulation of the primary motor cortex, after shaving and cleaning the skin with alcohol, the anodal electrode will be placed in the M1 area (Cz), and the cathodal electrode (reference) will be positioned between areas Fp1 and Fp2 of the 10/20 International System of Electroencephalography [19]. A direct current of $2 \mathrm{~mA}$ intensity and good impedance, ramping up for $15 \mathrm{~s}$, and subsequently ramping down for $15 \mathrm{~s}$ according to international safety guidelines will be applied [20]. The tDCS will be applied using an electrical stimulator, namely the battery-powered MedSupply Neurostim ${ }^{\circledR}$.

A total of 15 sessions will be performed using a schedule of three times a week for 5 weeks, wherein a blinded examiner will evaluate the participant before the beginning of the first session and 1 day after the last session. For the control group (sham mode), patients will be submitted to the same procedure, but the current will be shut off after $15 \mathrm{~s}$. The RT training protocol will be delivered during tDCS and will be composed by kicking a ball from a platform, placed either on the right or on the left side, depending on which light is turned on, for 10 min (between minute 5 until minute 15 from the 20 minutes of stimulation) (Figure 2).

The protocol will include physiologic monitoring of blood pressure, heart rate, respiratory rate, and peripheral oxygen saturation. The individual will also be instructed to stop the intervention if any discomfort is felt or if major hemodynamic changes occurs. The individuals will be discontinued if they present severe headache or epileptic seizure, and have no adaptation to the proposed protocol. No other type of intervention will be allowed during the trial.

\section{Primary outcome measures}

Change in the choice RT will be measured as follows: (1) as the RT in the upper limbs, and (2) as the RT in the lower limbs.

\section{Secondary outcome measures}

Changes in the scores of the scales will be measured as follows: (1) Trail Making Test and (2) Digit Span Test.

\section{Adverse effects}

All adverse effects during the study period will be reported using the safety questionnaire proposed by Brunoni et al. [21]. This process will be independent from investigators and the sponsor. 


\section{Participant timeline}

The enrollment, intervention, and assessment processes are displayed in Table 1.

Table 1. Enrollment, intervention, and assessment processes;

\begin{tabular}{|c|c|c|c|c|c|}
\hline \multirow[b]{3}{*}{ TIMEPOINT** } & \multicolumn{5}{|c|}{ STUDY PERIOD } \\
\hline & \multirow{2}{*}{$\begin{array}{l}\text { Enrollment } \\
-t_{1}\end{array}$} & \multirow{2}{*}{$\begin{array}{l}\text { Allocation } \\
0\end{array}$} & \multicolumn{2}{|c|}{ Post-allocation } & \multirow{2}{*}{$\begin{array}{l}\text { Close-out } \\
T_{16}\end{array}$} \\
\hline & & & $t_{1}$ & $t_{15}$ & \\
\hline \multicolumn{6}{|l|}{ ENROLLMENT: } \\
\hline Eligibility screen & $\mathrm{x}$ & & & & \\
\hline Informed consent & $\mathrm{x}$ & & & & \\
\hline Allocation & $x$ & & & & \\
\hline \multicolumn{6}{|l|}{ INTERVENTIONS: } \\
\hline$R T T$ & & & $x$ & $\mathrm{x}$ & \\
\hline Anodal tDCS & & & $\mathrm{x}$ & $\mathrm{x}$ & \\
\hline Sham tDCS & & & $x$ & $x$ & \\
\hline \multicolumn{6}{|l|}{ ASSESSMENTS: } \\
\hline$D S T$ & & $x$ & & $\mathrm{x}$ & $\mathrm{x}$ \\
\hline TMT & & $\mathrm{x}$ & & $\mathrm{x}$ & $\mathrm{x}$ \\
\hline$R T$ & & $x$ & & $\mathrm{X}$ & $x$ \\
\hline
\end{tabular}

$R T$, reaction time; RTT, reaction time training; tDCS, transcranial direct current stimulation; DST, Digit Span Test; TMT, Trail Making Test; T1 to T15 = period of intervention; T16 = next day (after $24 \mathrm{~h}$ )

\section{Baseline assessments}

\section{Choice Reaction time evaluation}

a) Upper limb: The participants will be seated in a chair with an adjustable height with their hips, knees, and ankles at $90^{\circ}$ of flexion, their shoulders between $10^{\circ}$ and $15^{\circ}$ of flexion, their elbows between $75^{\circ}$ and $90^{\circ}$ of flexion, and their forearms pronated. To avoid compensatory movements, the trunk will be stabilized at the $7^{\text {th }}$ thoracic vertebra. A monitor will be placed in front of the individual at a distance dependent on the upper limb length. The seat height will be adjusted to $100 \%$ of the lower limb length, which is measured from the lateral knee joint to the floor in a straight line with the subject standing barefoot. The center of the monitor height will be adjusted to $75 \%$ of the subject's shoulder height, which 
is defined as the distance from the shoulder marker to the floor with the subject sitting in the standardized position. The subject will have to reach to the ipsilateral or contralateral spaces in response to the visual stimulus, as quickly and accurately as possible, and return to the initial position at the end of the stimulus. The visual stimulus, represented by a white circle, lasts $5 \mathrm{~s}$ and can appear at five, six, seven, eight, or nine seconds in a randomized sequence (Figure 3).

b) Lower limb: the subjects will be positioned inside the platform and between two balls, located in front of a monitor, at a distance dependent on the lower limb length (the short the length, closer to the balls) (Figure 4). The individuals will have to kick the ball into the ipsilateral or contralateral spaces in response to a visual stimulus, as quickly and accurately as possible, and return to the initial position at the end. The visual stimulus, represented by a white circle, lasts $5 \mathrm{~s}$ and can appear at five, six, seven, eight, or nine seconds in a randomized sequence. The reaction time of the lower limbs will be measured before the upper limbs.

Both choice RTs will be evaluated by electromyographic signal according to the onset of the anterior deltoid, brachial biceps, triceps for upper limbs and rectus femoris, vastus medialis, and biceps femoris for the lower limbs. The EMG signals will be recorded using a Delsys Trigno TM® wireless telemetry sensor at 2,000 Hz according to the SENIAM protocol (Surface Electromyography for Noninvasive Assessment of Muscles) [22].

A photodiode will be used to synchronize the EMG signal with the visual stimulus. The RT in milliseconds will be calculated using the difference between the photodiode signal and EMG activity in the upper limb during reaching tasks and the kicking ball task.

\section{Evaluation of cognitive function}

Trail Making Test: This test consists of 25 circles distributed on a sheet of paper. In Part A, the circles are numbered $1-25$, and the patient must draw lines to connect the numbers in ascending order. In Part $B$, the circles include two numbers $(1-13)$ and letters $(A-L)$; as in Part $A$, the patient draws lines to connect the circles in an ascending pattern, but with the additional task of switching between numbers and letters (i.e., 1-A-2-B-3-C and so on). The patient will be instructed to connect the circles as quickly as possible without lifting the pen or pencil from the paper, marking the time with a chronometer that the individual takes to connect the circles [23].

Digit Span Test: This test consists of eight series for the direct order and seven for the reverse order, with a gradual increase in the number of digits in each series. The direct order is applied first, followed by the inverse, which is administered independently if the examinee completely fails in the direct order. Each item is made up of two sets of digits constituting two attempts, both of which are applied. The maximum score on the test is 30 points. The data analysis procedures include: 1) the calculation of the medians and the means of the raw scores to survey the performance standards of the normative samples as a function of age; 2 ) in each order, the maximum number of digits memorized by each subject of both samples will be identified, according to the procedure cited by Kaplan, Fein, Morris and Delis (1991), and 
subsequently the respective percentages calculated; 3) the discrepancies between the performance in the direct and reverse order will be calculated using the difference between the maximum number of digits correctly repeated in each order; and 4) when analyzing the discrepancies, it is also important to observe whether they are usual or unusual. For this reason, it becomes relevant to analyze how often the observed difference occurs in the standardization sample [24].

\section{Data analysis}

For data management, double data entry to promote data quality. To detect changes in continuous outcomes, a comparison between the anodal tDCS and the sham mode as well as between the tDCS and sham stimulation, will be performed using a t-test if a normal distribution is observed or the nonparametric Mann-Whitney test if no normal distribution is observed. The multiple imputation will be used if missing data. Data will be analyzed using SPSS version 22 (SPSS Inc., Chicago, IL 60606, USA), and will be considered statistically significant at a $p$ value $<0.05$.

\section{Discussion}

The FAST trial is a proposal that uses a novel method for the evaluation and training of choice RTs. The novelty is the combination of a specific functional activity of an athlete with a measurement of the choice RT necessary during a soccer game. Tanaka (2009) is the only study that utilized tDCS and reaction time training on the lower limbs, but their findings showed no significant difference in the RT after anodal tDCS and RT training [16]. Our study will use the online anodal tDCS in the same M1 area, during RT training in a platform developed by the authors to simulate kicking, instead of using pedals with seated patients. It is expected that RT training during the anodal tDCS stimulation will enhance the decrease in choice RT in soccer athletes and improve cognition function.

The anodal tDCS in conjunction with choice RT training with a functional task can be an efficient tool for athletes to prevent injuries. Our findings may be useful for soccer athletes to have a better and faster response during a game, and to improve performance in championships.

\section{Trial Status}

Protocol version number and data: $1(01 / 16 / 2020)$

Date recruitment began: 03/01/2020

Approximate date when recruitment will be completed: 03/01/2022

\section{Abbreviations}

RT

reaction time; tDCS:transcranial direct current stimulation; CONSORT:Consolidated Standards of Reporting Trials; HAD:Hospital Anxiety and Depression Scale; EMG:electromyography; SENIAM:Surface 
Electromyography for the Noninvasive Assessment of Muscles.

\section{Declarations}

\section{Acknowledgements}

The investigators would like to acknowledge the Federal University of Triângulo Mineiro for supporting this trial.

\section{Authors' contributions}

GJL and LAPSS were responsible for the project inception. EMN, GJL and LAPSS were involved in the study design, protocol preparation, drafting of the manuscript, and acquisition of funding. GJL and LAPSS conceived the statistical analyses and sample size calculation. EMN has been involved in merging the data from the principal FAST trial. EMN, GJL, and LAPSS prepared protocol details. All authors have reviewed and approved the final manuscript of the protocol.

Funding: The FAST trial will not have external sources of funding. This trial will be conducted without external funding and will be instead funded by the researchers' resources.

Availability of data and material: The principal investigator access to the final trial dataset.

Ethics approval and consent to participate: This trial was approved by the Committee for Ethics in Research involving human subjects from the Federal University of Triângulo Mineiro (CAAE: 23422619.4.0000.5154). The informed consent will be obtained from all study participants. Any modification to the protocol will be communicated to the registry of clinical trials and the ethics committee.

Consent for publication: "Not Applicable"

Competing interests: The authors declare that they have no competing interests.

Confidentiality: The collected data will be stored in locked and secure cabinets that will be accessible only to the physiotherapist who will perform the evaluations. Soon afterwards, the data will be input in a computer and saved and protected by a password to guarantee the confidentiality of the research participants' data. Any protocol modifications will be reported to the Ethics Committee and ReBEC.

Provisions for post-trial care: The participants can be compensated with post-trial care in Hospital of Federal University od Triângulo Mineiro if to those who suffer harm from trial participation.

Dissemination plans: via publication, reporting in results databases, or other data sharing arrangements.

\section{References}


1. Baker J, Cote J, Abernethy B. Learning from the experts: Practice activities of expert decision makers in sport. Res Q Exercise Sport. 2003;74:342-7.

2. North JS, Williams AM, Hodges N, Ward P, Ericsson KA. Perceiving patterns in dynamic action sequences: Investigating the processes underpinning stimulus recognition and anticipation skill. Appl Cogn Psychol. 2009;23:878-94.

3. Gorman AD, Abernethy B, Farrow D. Is the relationship between pattern recall and decision-making influenced by anticipatory recall? Q J Exp Psychol. 2013;66:2219-36.

4. Gorman A D, Abernethy B, Farrow D. Evidence of different underlying mechanisms in pattern recall and decision-making. Q J Exp Psychol. 2015;68:1813-31.

5. Savelsbergh G, Kamp J, Williams A, Ward P. Anticipation and visual search behavior in expert soccer goalkeepers. Ergonomics. 2005;15:1686-97.

6. Haywood KM, Getchell N. Desenvolvimento motor ao longo da vida. 3 ed. Porto Alegre: Artmed, 2004.

7. Ando S, Kida N, Oda S. Central and peripheral visual reaction time of soccer players and nonathletes. Percept Mot Skills. 2001;92:786-94.

8. Wilkerson GB, Simpson KA, Clark RA. Assessment and training of visuomotor reaction time for football injury prevention. J Sport Rehabil. 2017;26(1):26-34.

9. Molero-Chamizo A, Alameda BJR, Garrido BT, García LM, Jaén RI, Gutiérrez LC, Pérez PS, González ÁG, Lemus CL, Ruiz VMJ, Nitsche MA, Rivera-Urbina GN. Poststimulation time interval-dependent effects of motor cortex anodal tDCS on reaction-time task performance. Cogn Affect Behav Neurosci. 2018;18(1):167-75.

10. Poreisz C, Boros K, Antal A, Paulus W. Safety aspects of transcranial direct current stimulation concerning healthy subjects and patients. Brain Res Bull. 2007;72:208-14.

11. Nitsche MA, Paulus W. Excitability changes induced in the human motor cortex by weak transcranial direct current stimulation. J Physiol. 2000;527(Pt 3):633-9.

12. Hummel FC, Voller B, Celnik P, Floel A, Giraux P, Gerloff C, Cohen LG. Effects of brain polarization on reaction times and pinch force in chronic stroke. BMC Neurosci. 2006;7:73. [PubMed: 17083730]

13. Kang EK, Paik NJ. Effect of a tDCS electrode montage on implicit motor sequence learning in healthy subjects. Exp TransI Stroke Med. 2011;3:4. [PubMed: 21496317]

14. Leite J, Carvalho S, Fregni F, Goncalves OF. Task-specific effects of tDCS-induced cortical excitability changes on cognitive and motor sequence set shifting performance. PLoS One. 2011;6:e24140. [PubMed: 21909415]

15. Michael AN, Leonardo GC, Eric MW, Alberto P, Nicolas L, Andrea A, Walter P, Friedhelm H, Paulo SB, Felipe F, Alvaro PL. Transcranial direct current stimulation: state of the art 2008. Brain Stimul. 2008;1:206-23. [PubMed: 20633386]

16. Tanaka S, Takeda K, Otaka Y, et al. Single session of transcranial direct current stimulation transiently increases knee extensor force in patients with hemiparetic stroke. Neurorehabil Neural 
Repair. 2011; 25:565-9. doi: 1545968311402091 [pii]10.1177/1545968311402091. [PubMed: 21436391]

17. Seidel $O$, Ragert P. Effects of transcranial direct current stimulation of primary motor cortex on reaction time and tapping performance: a comparison between athletes and non-athletes. Front. Hum. Neurosci. 2019;13:103. doi: 10.3389/fnhum.2019.00103

18. Moher D, Schulz KF, Altman DG, for the CONSORT Group. The CONSORT Statement: revised recommendations for improving the quality of reports of parallel-group randomized trials. Lancet 2001;357:1191-4.

19. Jasper, Herbert $H$. "Report of the committee on methods of clinical examination in electroencephalography". Electroencephalogr Clin Neurophysiol. 1958;10 (2):370-5. doi:10.1016/0013-4694(58)90053-1.

20. Bikson M, Grossman P, Thomas C, et al. Safety of transcranial direct current stimulation: evidence based update 2016. Brain Stimul. 2016;9(5):641-61. doi:10.1016/j.brs.2016.06.004

21. Brunoni AR1, Amadera J, Berbel B, Volz MS, Rizzerio BG, Fregni F. A systematic review on reporting and assessment of adverse effects associated with transcranial direct current stimulation. Int $\mathrm{J}$ Neuropsychopharmacol. 2018;18(1):167-75.

22. Hermens JH, Freriks B, Klug CD, Rau G. Development of recommendations for SEMG sensors and sensor placement procedures. J Electromyogr Kinesiol. 2000;14:361-74.

23. Gaudino EA, Geisler MW, Squires NK. Construct validity in the Trail Making Test: what makes Part B harder? J Clin Exp Neuropsychol. 1995;17(4):529-35.

24. Reynolds CR. Forward and backward memory span should not be combined for clinical analysis. Arch Clin Neuropsychol. 1997;12(1):29-40. doi: 10.1016/S0887-6177(96)00015-7

\section{Figures}




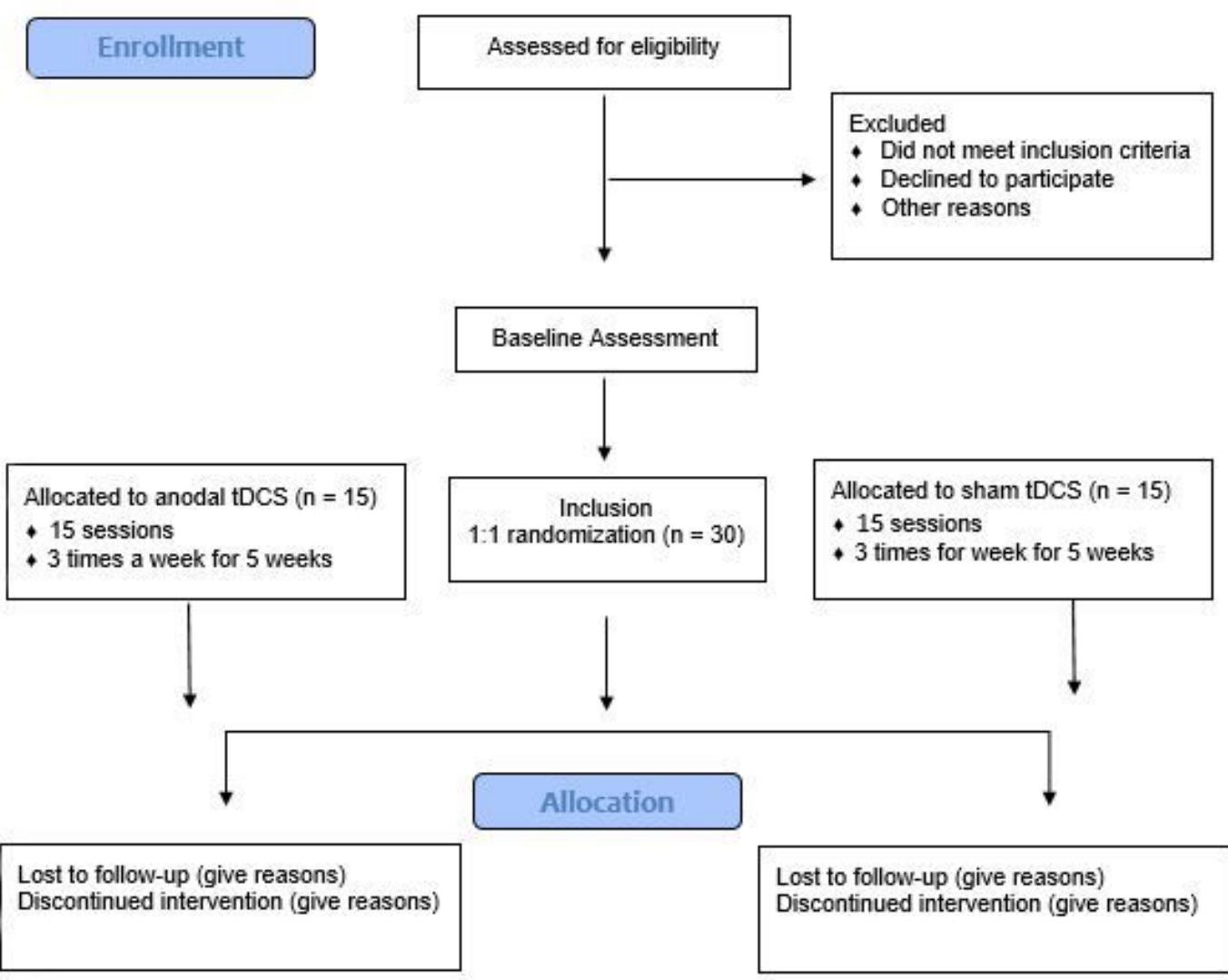

\section{Follow-Up}

Follow-up after intervention

- Trail Making Test

- Digit Span Test

- cognition function

Follow-up after intervention

- Trail Making Test

- Digit Span Test

- cognition function

\section{Figure 1}

Study Flow Diagram 

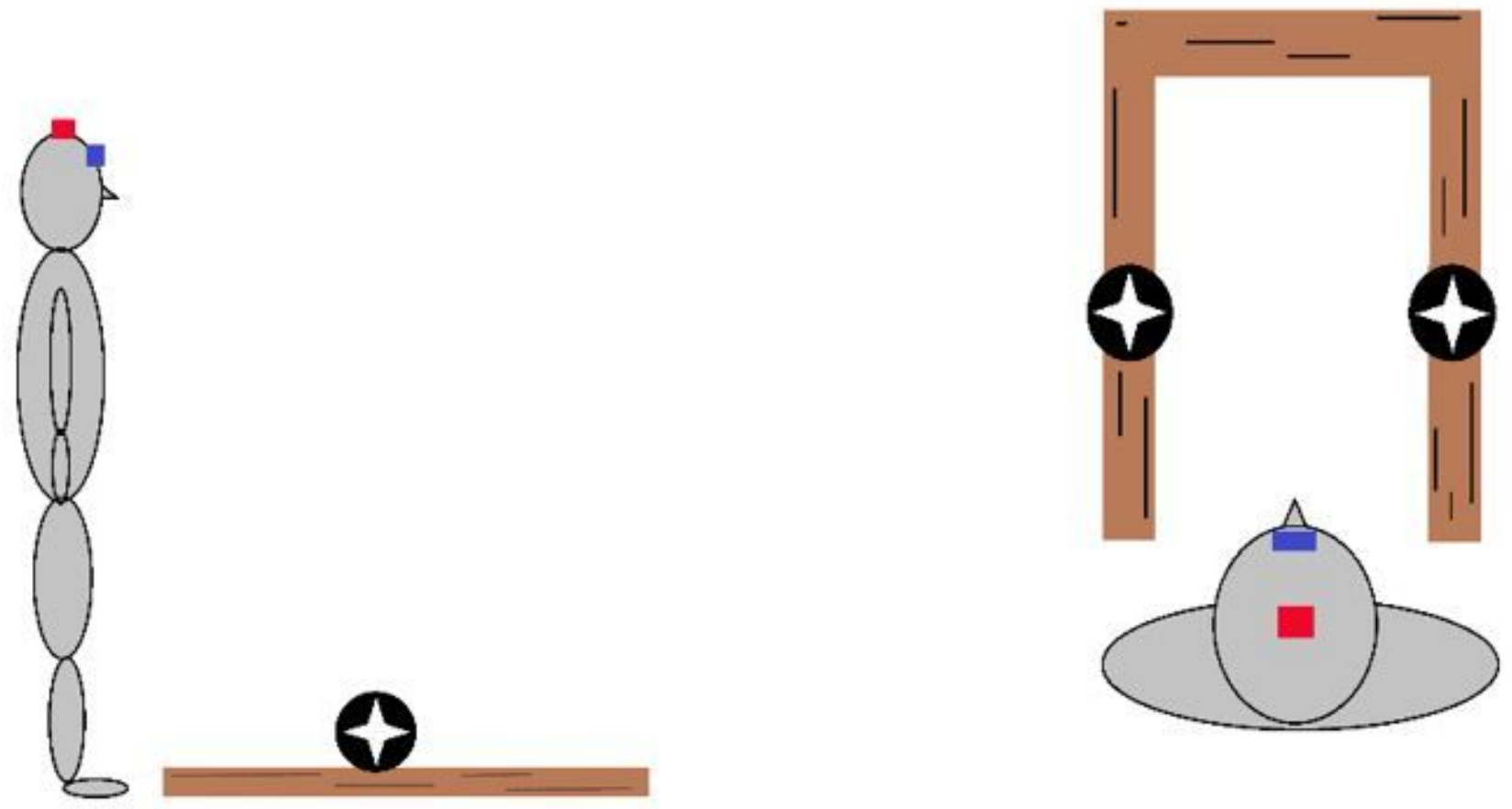

Figure 2

Participants receiving the anodal stimulation during the reaction time training. 


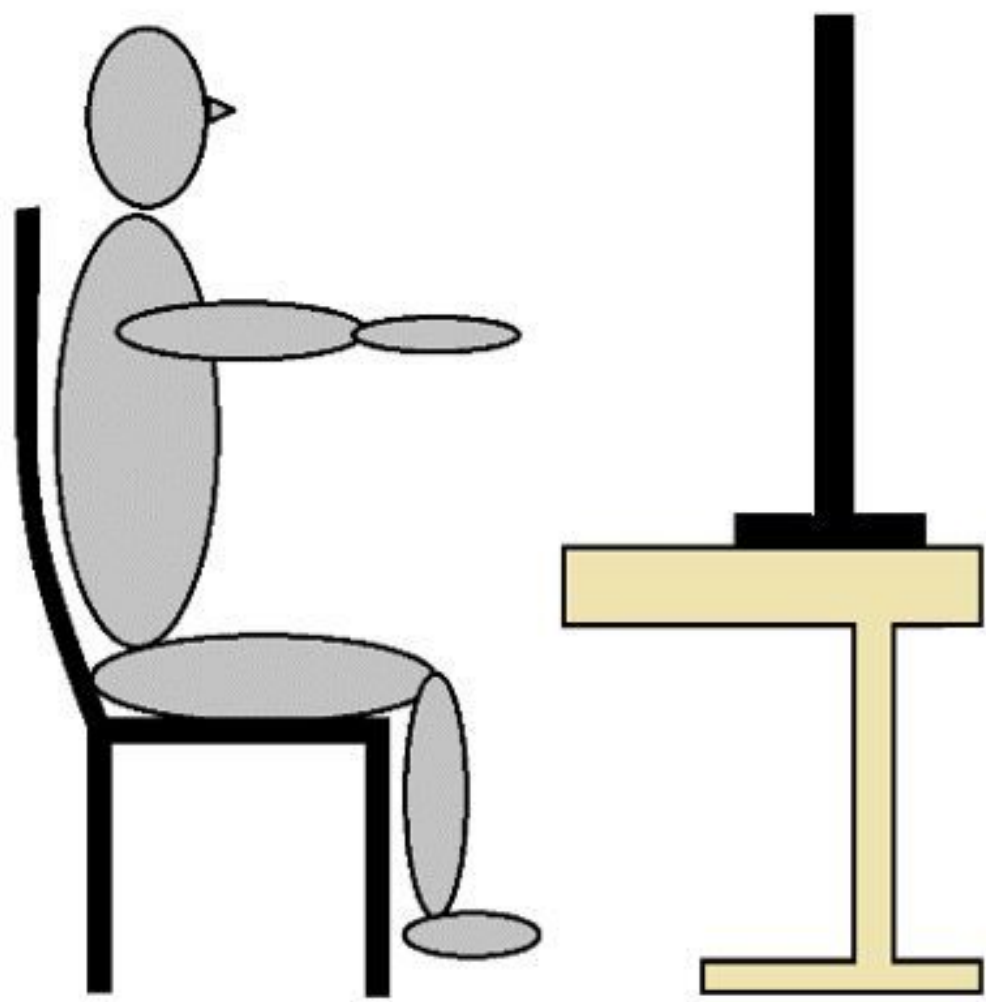

Figure 3

Evaluation of the choice reaction time in the upper limbs. 
A
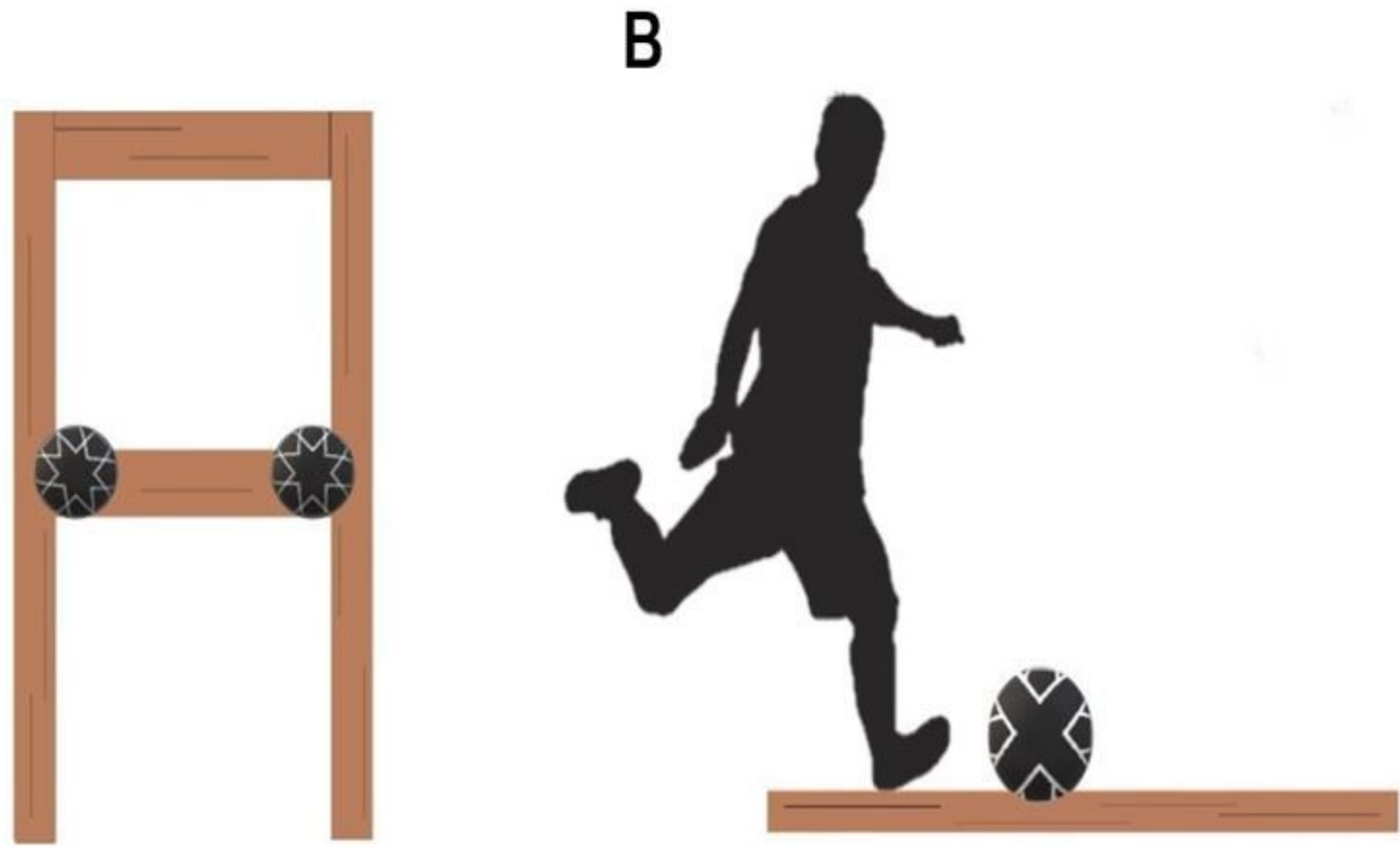

Figure 4

Evaluation of the choice reaction time in the lower limbs during the kick.

\section{Supplementary Files}

This is a list of supplementary files associated with this preprint. Click to download.

- Appendices.docx

- SPIRITChecklist.doc 